\title{
Significance of Urinary Plasmin in Patients with Systemic Lupus Erythematosus M.S.Eldein ${ }^{1}$, A.F.Soliman ${ }^{1}$, S.A.Mojahed ${ }^{1}$, S.K.Eliwa ${ }^{2}$ and R.M.Fawzy ${ }^{1}$ \\ ${ }^{1}$ Rheumatology, Rehabilitation and Physical medicine, Dept., Faculty of Medicine, Benha Univ., Benha, Egypt \\ ${ }^{2}$ Medical Biochemistry, Dept., Faculty of Medicine, Benha Univ., Benha, Egypt \\ Email:samia.mojahed@gmail.com
}

\begin{abstract}
Multisystem autoimmune disease SLE, which affects almost all organs and tissues, is a classic example of this kind of illness. Some researchers believe that because of the illness's great variability, SLE should be considered a syndrome rather than a single disease. In this study, the goal is to see whether urine plasmin levels may be used as a simple test and readily accessible marker in individuals with systemic lupus erythematosus. In this research, there were 50 SLE patients and 20 healthy volunteers. Urine samples were obtained and plasmin level was tested. Results showed that in SLE patients, urine plasmin levels were substantially higher than in healthy controls. Final thoughts: Urine-based biomarkers for detecting SLE patients are promising since they are noninvasive, safe, and helpful.
\end{abstract}

Keyword: Urinary Plasmin, Systemic Lupus Patients.

\section{Introduction}

SLE, or systemic lupus erythematosus, is a widespread, heterogamous autoimmune illness. When lupus manifests itself clinically, it may affect many organs, ranging from the musculoskeletal system to the skin to the mucous membranes to the blood cells to the brain and kidneys [1].

SLE is primarily caused by inflammation in combination with a lack of B-cell tolerance and the existence of B cells that are overactive, which allows the plasma cells to generate antinuclear antibodies (ANAs) (anti-nuclear antibodies). Endosomal Toll-like receptors are activated in SLE, as are immune complexes containing nucleic acid auto-antigens. Autoantibody production and intrarenal immune complex development characterise Lupus Nephritis (LN), which is a common sequela of SLE [3], [4]. Lupus Nephritis (LN) is an immune complex glomerulonephritis.

Autoantibodies have autoreactive plasma cells in the kidney [5]. SLE is linked to a high level of unmet demand and a significant impact on the quality of life of Egyptian lupus patients

Most SLE patients will suffer renal failure, cardiovascular problems, and mortality as a result of SLE nephritis [7]. In Egyptian SLE patients, frequent "pulse" steroid administration was linked to increased kidney damage, as well as adverse effects on the central nervous system and bones [8]. For this susceptible rheumatic illness, persistent national efforts are being made to improve clinical practise standards, optimise the use of biologics, as well as create customised and targeted treatments [5].

There is still a pressing need for easy-to-measure biomarkers. If urine indicators of kidney damage can be detected, it will have a significant impact on how disease activity and inflammation are assessed.

Phosphorylation of its precursor, the 810aglycoprotein plasminogen, results in the production of the serine protease, plasmin, in the body. Plasma plasminogen concentrations reach $2.4 \mathrm{M}$ after cleavage of a 19-aa signal peptide [10].

\section{Aim of the work}

To evaluate urine plasmin level as an easy test and available marker in systemic lupus erythematosus (SLE) patients.

\section{Patients and methods}

Fifty SLE patients fulfilled the classification criteria for Systemic Lupus International Collaborating Clinics (SLICC) (2012) [11] and 20 matched controls for age and sex were included. Urine plasmin level was measured using enzyme linked immunosorbent technique (ELISA) technique. The study was approved by ethical committee of Benha University.

History from all SLE patients was obtained and clinical examination was performed. Routine laboratory investigations were ordered and urine samples were collected to measure urine plasmin level.

Patients with acute/chronic infection, diabetes mellitus, other autoimmune diseases and end stage organ failure were excluded.

\subsection{Statistical analysis}

Data were coded and entered using the statistical package SPSS (Statistical Package for the Social Science; SPSS Inc., Chicago, IL, USA) version 26. Data was summarized using number $\&$ percentage and mean, standard deviation ( mean \pm SD). P-value less than 0.05 was considered as significant and less than 0.001 highly significant.

\section{Results}

This study included 50 SLE patients together with 20 age and sex matched healthy controls.

No significant differences were reported between the studied groups as regard age and sex distribution (p>0.05). 
Table (1) shows clinical examination findings among the studied SLE cases.

\begin{tabular}{lcc}
\hline & & No(\%) \\
\hline Mucocutaneous manifestations & 45 & $(90)$ \\
Eye & 7 & $(14)$ \\
Arthritis & 5 & $(10)$ \\
Renal manifestations & 28 & $(56)$ \\
Pulmonary manifestation & 12 & $(24)$ \\
Cardiac manifestations & 7 & $(14)$ \\
CNS manifestations & 6 & $(12)$ \\
\hline
\end{tabular}

Regarding mean urine plasmin levels, significant difference $(p<0.001)$ was observed between the studied groups being higher among SLE cases.

As regard medications received by SLE cases; there were 29 cases $(58.0 \%)$ on regular Corticosteroids, while all patients were on regular immunosuppressive drugs.

\section{Discussion}

Lymphoma (also known as SLE or Lupus) is the prototypic systemic autoimmune disease with widespread involvement of many organ systems and an array of autoantibodies [12]. One-third of SLE patients develop lupus nephritis (LN) as their first symptom [13].

Glomerular autoantibody deposition and other immune complex or complements lead to the generation of proinflammatory cytokines as well as the infiltration of immune cells. These events lead to cell proliferation as well as vascular rarefaction injuries. These injuries can harm the kidneys through multiple mechanisms, eventually leading to tubulointerstitial fibrosis and glomerulo-sclerosis [14] (see below).

SLE patients had higher urine plasmin levels than healthy controls, according to the results of the present research. According to these results, SLE has an effect on renal function.

Since renal pathology may change over time, having a real-time readout of kidney function might be beneficial for developing a treatment strategy for LN and monitoring the progress of the patient. Therefore, a therapeutically relevant objective is to identify urine biomarkers that correctly represent kidney disease during LN flare cycles. Urine biomarkers that identify $\mathrm{LN}$ from other types of glomerular illness or that can discriminate between LN classes[15] have been sought in certain studies to address this issue.

If effective, these biomarkers will provide a comprehensive picture of renal disease by reflecting the total amount of kidney damage.

It was suggested in a previous article that the plasminogen activator system evolved from a single common gene, which then duplicated and diverged into the plasminogen activator system proteins. HGF, lipoprotein(a), and macrophage stimulating 1 have evolved similarly to plasminogen, whereas hyaluronanbinding protein, and hepatocyte growth factor activator are evolutionarily comparable to tPA and uPA, the scientists found. Similar to plasminogen, these related proteins have a variety of activities, but they all have a connection to the vasculature and inflammation.

Coagulation and innate immune proteins developed together to regulate these physiological processes [18], which may explain the increased amount of urine plasmin in SLE patients. Inflammatory proteins are mediators of coagulation processes.

\section{Conclusion}

For the identification of people with systemic lupus erythematosus, urine plasmin may be a valuable, safe, and noninvasive biomarker.

\section{References}

[1] C. Putterman, R. Caricchio, A. Davidson, H. Perl man. Systemic lupus erythematosus Clin Dev Immunol, Article 437282, 2012.

[2] A.M. Fairhurst, A.E. Wandstrat, E.K. WakelandS ystemic. lupus erythematosus: multiple immunological phenotypes in a complex genetic disease Adv. Vol. 92 , pp. 1-69, 2006.

[3] M. Lech, H. Anders. The Pathogenesis of lupus nephritis. J Am Soc Nephrol. Vol. 9, pp. 13571366, 2013.

[4] Y. Liu, H. Anders. Lupus nephritis: from pathogenesis to targets for biologic treatment Nephron Clin Pract. Vol. 3-4, pp. 224-231, 2014.

[5] M. Espeli, S. Bökers, G. Giannico, H.A. Dickinso n, V. Bardsley, A.B. Fogo, et al. Local renal autoantibody production in lupus nephritis $\mathrm{J}$ Am Soc Nephrol. Vol. 2 , pp. 296-305, 2011.

[6] N.M. Gaballah, A.R. El-Najjar:Clinical characteristics and health related quality of life (HRQoL) in Egyptian patients with systemic lupuserythematosus Egyptian Rheumatol. Vol.41 (2), pp. 117-121, 2019.

[7] G. Bertsias, M. Tektonidou, Z. Amoura, M. Arin ger, I. Bajema, J.H. Berden, et al. Joint european league against rheumatism and European Renal Association-European Dialysis and Transplant Association (EULAR/ERA-EDTA) recommendations for the management of adult and paediatric lupus nephritis. Ann Rheum Dis. Vol. 71, pp. 1771-1782, 2012.

[8] A.M. Elhefny, H.M. Farouk, N.O. ElAzizi, A.A. Elzaher, E.S. Mohammed. Hazards of pulse steroid use in a cohort of Egyptian lupus 
nephritis patients Egypt Rheumatol. Vol. 41 (4), pp. 277-281, 2019.

[9] D. Sun, X. Zhao, L. Meng. Relationship between urinary podocytes and kidney diseases Ren Fail. Vol. 34 (3) , pp. 403-407, 2012.

[10] L. De Souza, P. Melo, T. Paschoalin, et al. Human tissue kallikreins 3 and 5 can act as plasminogen activator releasing active plasmin. Biochem Biophys Res Commun. Vol. 3(3), pp. 333-337, 2013.

[11] M. Petri, AM. Orbai, GS. Alarcón, et al. Derivation and Validation of Systemic Lupus International Collaborating Clinics Classification Criteria for Systemic Lupus Erythromatosus. Arthritis Rheum. Vol. 46(8), pp.2677-86, 2012.

[12] MI. Danila, GJ. Pons-Estel, J. Zhang, LM. Vilá, JD. Reveille, GS. Alarcón. Renal damage is the most important predictor of mortality within the damage index: data from LUMINA LXIV, a multiethnic US cohort Rheumatology (Oxford). Vol. 48 (5) ,pp. 542-545, 2009.

[13] GJ. Hanly ,A. O'Keeffe, L. Su, MB. Urowitz, J. Romero-Diaz, C. Gordon, et al. The frequency and outcome of lupus nephritis: results from an international inception cohort study Rheumatology (Oxford). Vol. 55 (2), pp. 252262, 2016.

[14] A. Davidson. What is damaging the kidney in lupus nephritis? Nat Rev Rheumatol. Vol. 12 (3) ,pp. 143-153, 2016.

[15] SA. Varghese, TB. Powell, MN. Budisavljevic, et al. Urine biomarkers predict the cause of glomerular disease. J Am Soc Nephrol. Vol. 18, pp. 913-922, 2007.

[16] JC. Oates, S. Varghese, AM. Bland, et al. Prediction of urinary protein markers in lupus nephritis. Kidney Int. vol. 68, pp. 2588-2592, 2005.

[17] A. Chana-Muñoz, A. Jendroszek, M. Sønnichsen, et al. Origin and diversification of the plasminogen activation system among chordates. BMC Evol Biol. Vol. 19, pp.20-27, 2019.

[18] S. Baker, S. A Strickland. critical role for plasminogen in inflammation. JEM. Vol.217(4), pp. $1-13,2020$. 